\title{
USO DO MÉTODO DO ELEMENTO DISCRETO DEM COMO APROXIMAÇÃO DO PROCESSO DE SEPARAÇÃO ELETROSTÁTICA*
}

José Raphael Olegário França ${ }^{1}$

Rodrigo Magalhães de Carvalho²

\section{Resumo}

Separação eletrostática é o termo usado para todas as tecnologias de processamento que utilizam forças elétricas que atuam sobre partículas carregadas ou polarizadas sob um campo elétrico estático. O comportamento das partículas depende das suas propriedades elétricas, geométricos e tamanho das partículas. Portanto, a separação de espécies é possível se os parâmetros do campo elétrico e as propriedades elétricas diferentes das partículas são apropriados para o processo. No separador eletrostático de rotor as forças elétricas, combinadas com as forças gravitacionais e centrífugas agem sobre as partículas e são responsáveis pela separação mineral. No presente trabalho as forças eletrostáticas que agem no processo serão substituídas por um modelo de contato de coesão Hertz-Mindlin com JKR. O resultado apresentado, embora com limitações, conseguiu-se fazer uma boa estimativa do processo e alguns dos parâmetros do separador. Com a presente simulação é possível descrever o processo de separação além de permitir a redução de despesas de testes práticos, melhorar a compreensão das relações fundamentais, simular as influências dos diferentes parâmetros e por fim, estimar eventuais aumento de desempenho.

Palavras-chave: Separação eletrostática de rotor; Método dos elementos discretos.

\section{USE OF DISCRETE ELEMENT METHOD DEM AS APPROXIMATION OF ELECTROSTATIC SEPARATION PROCESS}

\section{Abstract}

Electrostatic separation is the term used for all processing technologies that use electrical forces acting on charged or polarized particles in a static electric field. The behavior of the particles depends on their electrical properties, geometry and particle size. Therefore, the separation of species is possible if the parameters of the electric field and the different electrical properties of the particles are suitable for the process. In rotor electrostatic separator electrical forces coupled with the gravitational and centrifugal forces act on particles and are responsible for mineral separation. In this work the electrostatic forces that act in the process will be replaced by a cohesion contact Hertz-Mindlin model with JKR. The result presented, although with limitations, has managed to make a good estimate of the process and some of the separator parameters. With this simulation it is possible to describe the separation process and allows the reduction of practical testing costs, improve understanding of fundamental relationships, simulating the influences of different parameters and finally to estimate any performance increase.

Keywords: Rotor electrostatic separation; Method of discrete elements.

1 Mestrando em Eng. Metalúrgica, Universidade Federal do Rio de Janeiro, Rio de Janeiro, Rio de Janeiro, Brasil.

2 D.Sc. Professor Adjunto, Universidade Federal do Rio de Janeiro, Rio de Janeiro, Rio de Janeiro, Brasil. 


\section{INTRODUÇÃO}

Alguns dos princípios básicos de separação eletrostática têm sido bem conhecido desde tempos antigos. Thales de Mileto (600 a.c.) observou que uma haste de âmbar atraia partículas de poeira depois de ser esfregada com um pano. Processos eletrostáticos foram primeiramente utilizados por volta de 1880 para fins agrícolas na separação do joio do grão de trigo. Com o surgimento de fontes de altas tensões necessárias para o carregamento das partículas, outras aplicações tornou-se possível. Hoje em dia numerosas instalações industriais, processam milhões de toneladas de sais, minérios de ferro, minérios de titânio, minerais pesados de areias de praia entre outras áreas da indústria estão em operação, demonstrando o potencial desta tecnologia. [1-3]

Separadores eletrostáticos de rotor utilizam as diferenças de condutividade elétrica entre minerais que compõem o minério (Figura 1). Na prática, porém, o processo apresenta limitações que impedem sua ampla utilização. $O$ fato de que a alimentação deve ser perfeitamente seca impõe limitações no processo além de apresentar uma capacidade muito pequena para materiais finamente dividido. Para uma maior eficiência a alimentação deve estar numa única camada de profundidade, o que também limita severamente a produtividade se as partículas são muito pequenas, $75 \mathrm{um}$.

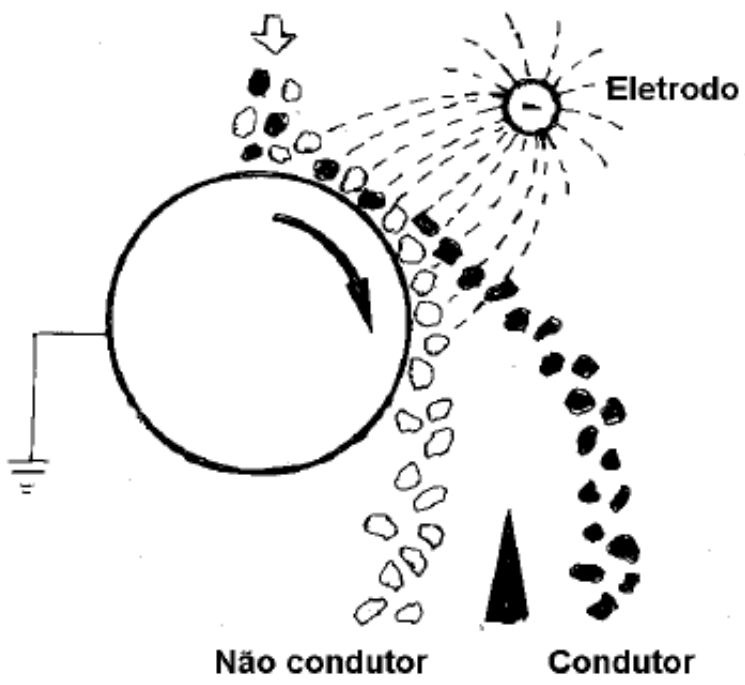

Figura 1. Equipamento de separação eletrostática de rotor. [2]

O tamanho da partícula influencia diretamente no comportamento de separação. Relacionando as cargas superficiais com o seu tamanho é sabido que em um grão grosso as cargas são menores em relação à sua massa que em um grão fino. Assim, um grão grosso é mais facilmente jogado a partir da superfície do cilindro, e a fração de partículas condutoras muitas vezes contém uma pequena proporção de partículas grossas não-condutores. De modo semelhante, as partículas mais finas são mais influenciadas pela carga de superfície e a fração não-condutora muitas vezes contém algumas partículas condutoras finas. [4-7]

Vale salientar que a demanda ambiental de processamento de minerais a seco torna-se um ponto importante o que é uma característica do processo de separação eletrostática. 


\subsection{Aspectos Teóricos}

O carregamento de uma partícula ocorre apenas na sua superfície, não dentro de toda a partícula. Portanto, um fator relevante para o carregamento é a superfície específica. A propriedade física mais importante é a condutividade da superfície de uma partícula. Uma superfície de alta condutividade irá facilitar o descarregamento de uma partícula. A constante dielétrica está relacionada diretamente com a facilidade de uma partícula adquirir cargas e assim mais fácil será a separação. Uma grande diferença entre as características dos materiais a serem separados favorece assim a sua separação.

A condição para uma partícula isolante de massa $\mathrm{m}$ ficar aderida ao elétrodo do cilindro metálico com um raio $\mathrm{R}$, que gira a uma velocidade angular $\omega$, pode ser expressa da seguinte forma (Equação 1):

$$
\mathrm{Fi}>\mathrm{Fc}-\mathrm{Fg} \cos \beta
$$

onde, Fi é a força de imagem, $\mathrm{Fc}=m \omega^{2} \mathrm{R}$ é a força centrífuga, e $\mathrm{Fg}=\mathrm{mg}$ é a força da gravidade que atua na partícula (Figura 2). Na equação acima $\beta$ representa a posição angular das partículas no cilindro. [1-6]

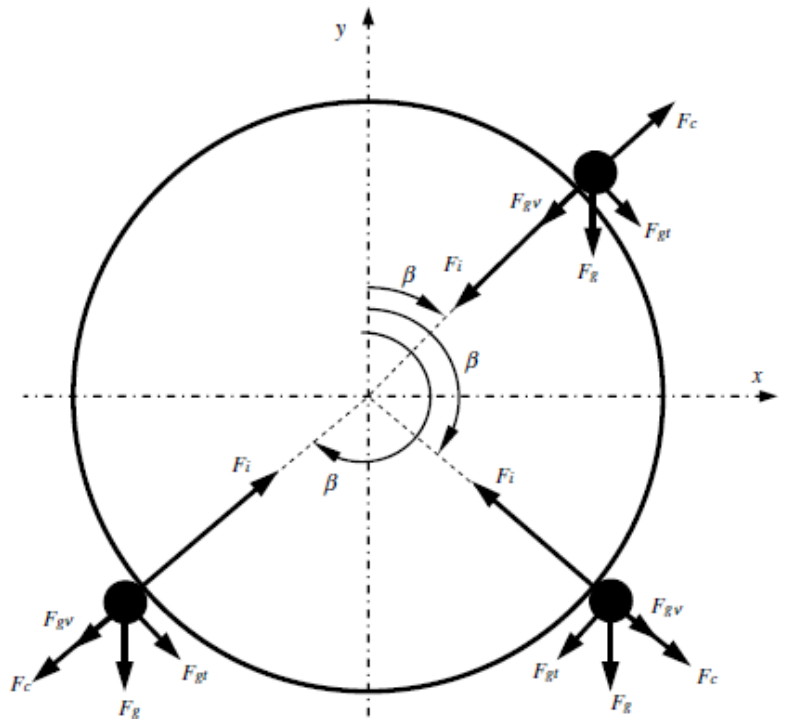

Figura 2. Forças no separador eletrostático de rotor. [4]

A força de imagem Fi sobre uma partícula esférica pode ser calculada com a seguinte fórmula (Equação 2):

$$
\mathrm{Fi}=Q^{2} /\left(16 \pi \varepsilon a^{2}\right)
$$

onde $Q$ é a carga instantânea da partícula, $\varepsilon$ é a permissividade do meio e $a$ é o raio da partícula (Figura 3). [4] 


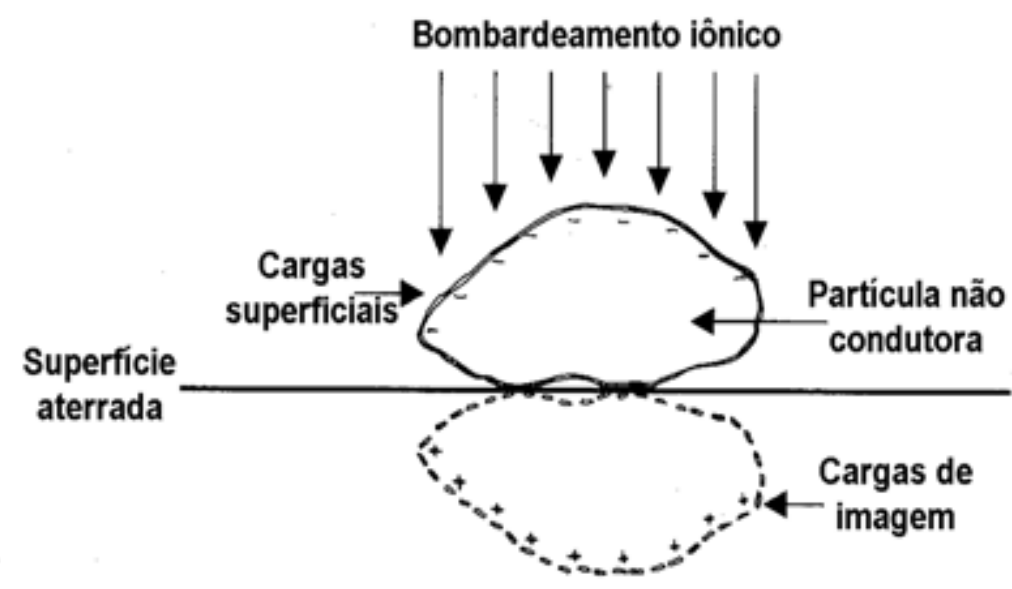

Figura 3. Forças de imagem numa partícula isolante. [2]

Do ponto de vista da separação de alta tensão o critério utilizado para distinguir partículas condutoras de isolantes é o tempo de relaxação, $\operatorname{Tr}$ (Equação 3). O $\operatorname{Tr}$ de uma partícula carregada é definido como o tempo necessário para que uma densidade de carga inicial po reduza-se a 1/e de seu valor original (Figura 4). Para partículas condutoras sua carga superficial rapidamente decai sendo lançadas do cilindro com baixos ângulos de rotação $\beta$, enquanto que partículas isolantes permanecem carregadas e seguem aderidas ao cilindro. [2-9]

$\rho_{0}=$ densidade de carga inicial

$$
\rho=\rho_{0} \exp (-\sigma t / \varepsilon)
$$

$\rho=$ densidade de carga no tempo $t$

$\sigma=$ condutividade da partícula

$\varepsilon=$ permicividade da partícula

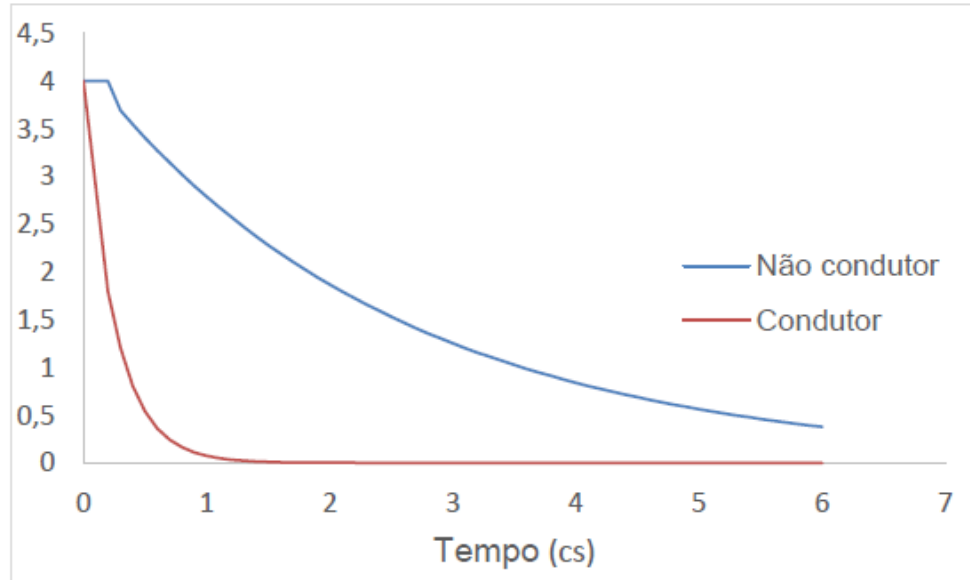

Figura 4. Tempo de relaxamento entre partículas condutoras e não condutoras.

Para a simulação do separador eletrostático de rotor foi utilizado um modelo de forças de coesão Hertz-Mindlin com JKR (Johnson-Kendall-Roberts). Este modelo de contato permite representar a natureza coesiva de materiais finos e úmidos que nesta simulação substitui a força de imagem entre as partículas e o cilindro. Originalmente implementado para permitir a simulação do comportamento do fluxo de pós finos secos influenciado por forças de Van der Waals. [7, 8] Este modelo não representa corretamente as forças que agem no processo de separação eletrostática 
nem as diferenças de propriedades elétricas das partículas ele foi escolhido apenas para ilustrar o processo.

\section{MATERIAIS E MÉTODOS}

Utilizou-se o programa EDEM 2.7.0 versão sala de aula para construir a geometria e a simulação do processo de separação eletrostática de cilindro. Para a simulação do separador eletrostático foi utilizado um modelo de forças Hertz- Mindlin com JKR (Johnson-Kendall-Roberts) de coesão.

Para a simulação foram criados dois tipos de partículas, uma representando o mineral de interesse condutor e a outra representando a ganga não condutora. Para a ganga foi utilizado o modelo de contato JKR entre a mesma e o rolo. Esta força de coesão representa a força de imagem que as partículas isolantes geram em seu dipolo induzido.

Para simplificar a simulação foi utilizado uma escala ampliada do sistema separador e partículas. As partículas de minério e ganga foram configuradas com diâmetros de $10 \mathrm{~mm}$ e $5 \mathrm{~mm}$, massas de $0,012 \mathrm{~kg}$ e $0,00084 \mathrm{~kg}$ respectivamente. O valor da energia superficial das partículas isolantes da ganga foi calibrada fazendo-se outra simulação. Na calibração a partícula era solta sob o cilindro com determinada energia superficial de modo a encontrar um valor razoável no qual a partícula permanecesse levemente aderida a superfície do cilindro. O valor encontrado foi de $30 \mathrm{~J} / \mathrm{cm}^{2}$, para valores inferiores a separação não se realizava (Figura 5).

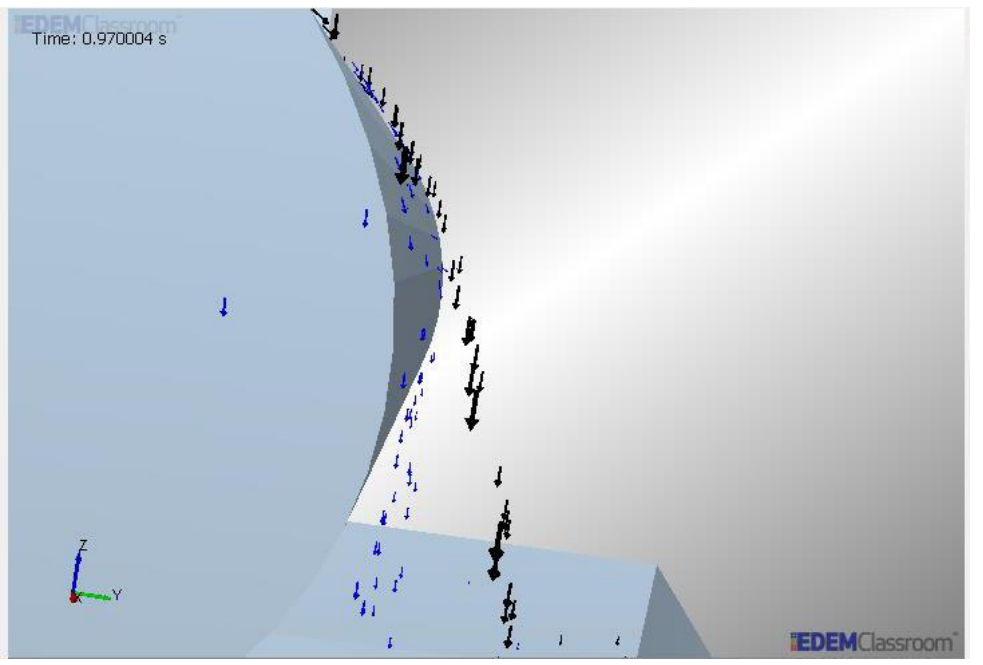

Figura 5. Simulação com energia superficial específica de $27 \mathrm{~J} / \mathrm{cm}^{2}$.

A rotação do cilindro foi configurada em 40 rpm o que fornece uma força centrífuga de sentido oposto à força de coesão entre a ganga e o cilindro.

\section{RESULTADOS E DISCUSSÃO}

A força centrífuga é proporcional ao quadrado da velocidade angular e diretamente proporcional a massa da partícula. Conforme os dados extraídos do EDEM a força que age no eixo z para as partículas de minério tem o módulo do valor de cerca de 23 vezes maior que a força que age nas partículas de ganga. De acordo com os resultados obtidos das forças que agem nas partículas $\mathrm{Fc}, \mathrm{Fg}$ e $\mathrm{Fi}$ e a condição de separação da equação (1) Fi>Fc-Fgcos $\beta$ ocorre a separação das partículas de 
minério e de ganga, partículas pretas representam o minério de interesse e as partículas em azul a ganga (Figura 6).

As partículas da ganga satisfazem a condição da (Equação 1) pois a força $\mathrm{Fi}$ de atração da partícula com o cilindro é superior à soma resultante das forças $\mathrm{Fc}$ e Fg. Isso resulta em uma aderência das partículas com a superfície do cilindro, partículas em azul (Figura 6).

Para as partículas de minério, elas não satisfazem a condição da (Equação 1) pois a soma das forças $\mathrm{Fc}$ e $\mathrm{Fg}$ são superiores a força de atração com o cilindro, Fi. Isso resulta no desprendimento das partículas da superfície do cilindro, partículas pretas (Figura 7).
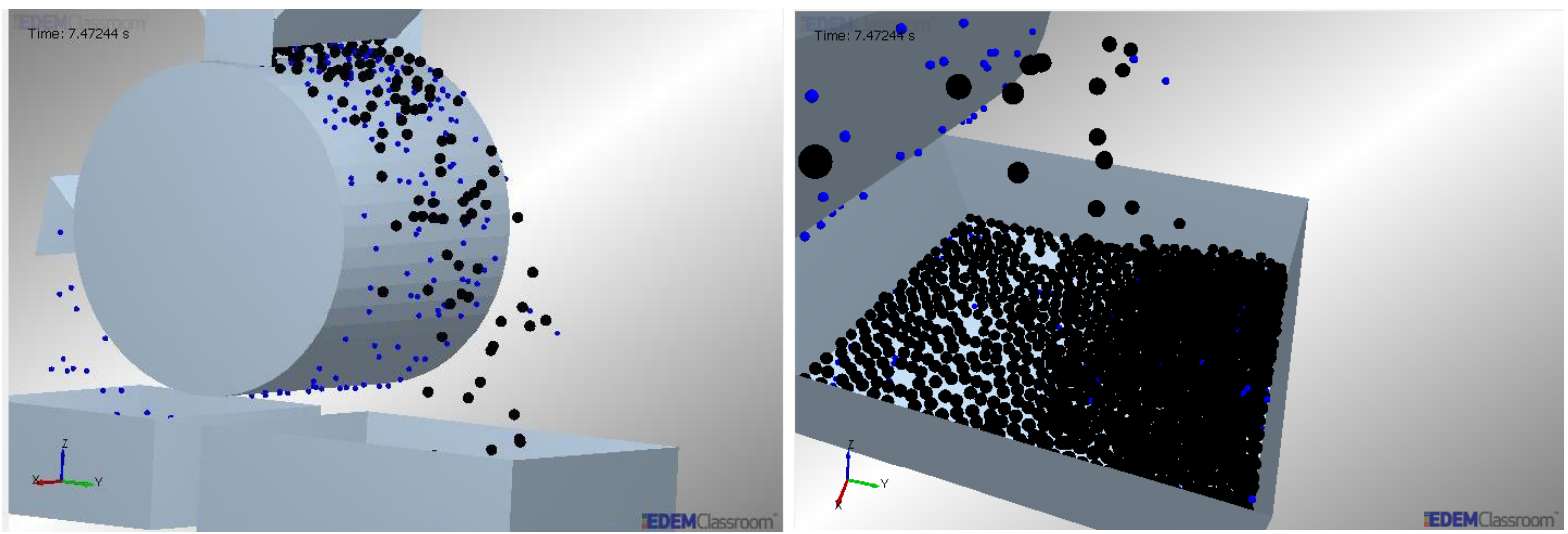

Figura 6 e 7. Processo de separação eletrostática e ocorrência de curto-circuito, respectivamente.

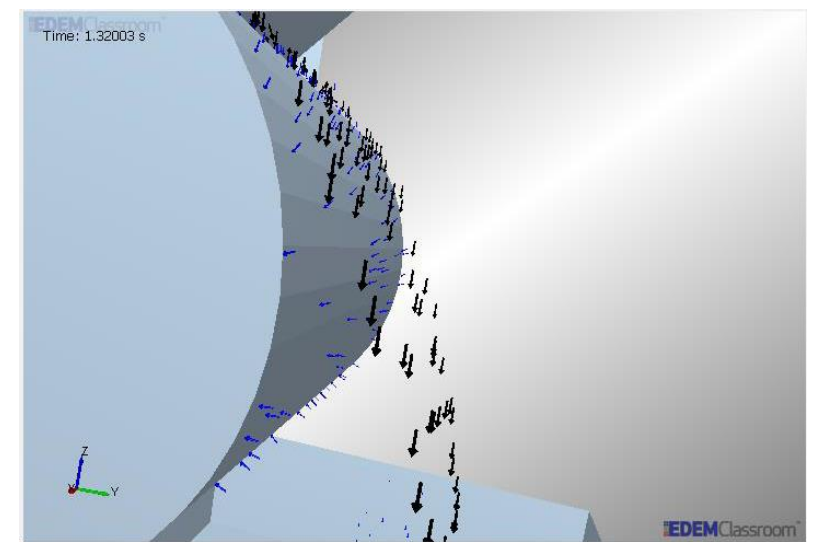

Figura 8. Vetor força resultante das partículas na separação.

Como pode ser observado nas figuras anteriores há partículas de ganga que reportam ao compartimento das partículas do minério de interesse. A possibilidade para esse fato é que as partículas não estão sendo devidamente alimentadas. $O$ excesso na alimentação causa a não separação das partículas que gera o curtocircuito. Para analisar melhor este fato foram extraídos os dados das forças médias na direção vertical z das partículas (Figura 8 e 9). 


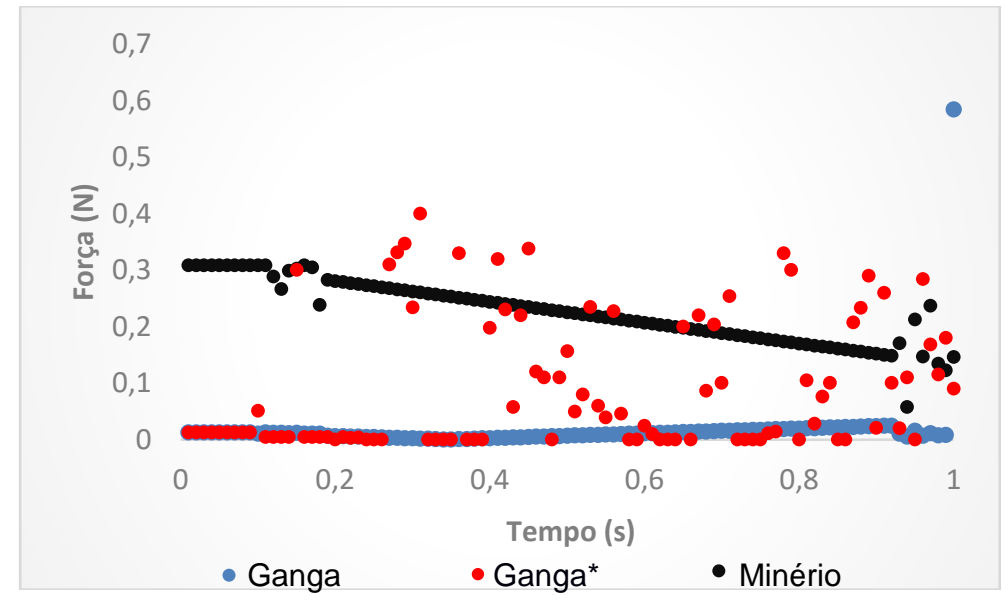

Figura 9. Força máxima ganga* e força média das partículas de minério e ganga.

Os pontos vermelhos são os dados das forças máximas em $z$ das partículas de ganga. Algumas partículas apresentaram forças capazes de se desprenderem do cilindro superando a força de imagem, sendo estas partículas as que se reportaram ao compartimento do minério de interesse. Outros pontos do gráfico superaram a força do minério o que se torna estranho pois a massa das partículas de ganga é bem inferior à do minério.

\section{CONCLUSÃO}

A separação eletrostática pelo processo separador de rotor é significativamente influenciada pelas propriedades das partículas tais como densidade, massa, tamanho, além da permissividade, condutividade das partículas e pelos parâmetros do equipamento como velocidade de rotação e força do campo elétrico, nesse caso a energia superficial de coesão do cilindro com as partículas.

Outro fator importante para uma boa separação é controlar bem a alimentação para que todas as partículas entrem em contato com o cilindro evitando que partículas sejam lançadas diretamente sem serem separadas evitando o curto-circuito do processo.

Por fim o método dos elementos discretos embora não possua as forças de corpo que simule as forças eletrostáticas, ele possibilita a implementação dessa grandeza. O modelo de coesão JKR utilizado em substituição à força de imagem se mostrou satisfatório permitindo que a presente simulação descreva bem o processo de separação, possibilitando a redução de despesas de testes práticos e por fim estimar eventuais aumento de desempenho.

\section{Agradecimentos}

Os autores que realizaram este trabalho gostariam de agradecer ao LTM Coppe/UFRJ (Laboratório de Tecnologia Mineral Universidade Federal do Rio de Janeiro) pelo fornecimento das ferramentas utilizadas nesse trabalho e a CAPES (Coordenação de Aperfeiçoamento de Pessoal de Nível Superior) pela concessão da bolsa de mestrado. 


\section{REFERÊNCIAS}

1 L. Brands, P. M. Beier, I. Stahl, Electrostatic Separation, Encyclopedia of Industrial Chemistry, Wiley-VCH Verlag GmbH, 2012.

2 L. M. Tavares, processamento de recursos minerais III, UFRJ - LTM, 2004.

3 E. G. Kelly, D. J. Spottiswood, The Theory of Electrostatic Separations: A Review Part I, II and III, Minerals Engineering, 1988.

4 S. Das, A. Samuila, C. Dragan, L. Dascalescu, Behaviour of charged insulating particles in contact with a rotating roll electrode, Journal of Electrostatic, 2009.

5 J. E. Lawver, State of the Art of Electrostatic Separation of Minerals, Journal of the Electrochemical Society, 1969.

6 Y. Higashiyama, K. Asano, recent progress in electrostatic separation technology, Particulate Science and Technology: An International Journal, 1998.

7 DEM Solutions, EDEM 2.7 User Guide, 2015 UK.

8 K. L. Johnson, K. Kendall, A.D. Roberts, Surface energy and the contact of elastic solids - JKR model, The Royal Society, 1971, 301-313.

9 L. Dascalescu, R. Morar, A. luga, A. Samuila, V. Neamtu, electrostatic separation of insulating and conductive particles from granular mixes, Particulate Science and Technology: An International Journal, 2013. 